\title{
GAUSSIAN ESTIMATES FOR FUNDAMENTAL SOLUTIONS TO CERTAIN PARABOLIC SYSTEMS
}

\author{
Steve Hofmann and Seick Kim
}

Abstract

Auscher proved Gaussian upper bound estimates for the fundamental solutions to parabolic equations with complex coefficients in the case when coefficients are time-independent and a small perturbation of real coefficients. We prove the equivalence between the local boundedness property of solutions to a parabolic system and a Gaussian upper bound for its fundamental matrix. As a consequence, we extend Auscher's result to the time dependent case.

\section{Introduction and Main result}

Consider $N \times N$ system of equations

$$
D_{t} u^{i}-\sum_{j=1}^{N} \sum_{\alpha, \beta=1}^{n} D_{x_{\alpha}}\left(A_{i j}^{\alpha \beta}(x, t) D_{x_{\beta}} u^{j}\right)=0 \quad(i=1, \ldots, N) .
$$

Here $t$ is a real number and $x=\left(x_{1}, \ldots, x_{n}\right) \in \mathbb{R}^{n}$. For each $\alpha, \beta=$ $1, \ldots, n$, we shall denote by $\boldsymbol{A}^{\alpha \beta}(x, t)$ an $N \times N$ matrix with $(i, j)$ entries of $A_{i j}^{\alpha \beta}(x, t)$.

It is convenient to write the system (1.1) in a vector form.

$$
\mathcal{L} \boldsymbol{u}:=\boldsymbol{u}_{t}-\sum_{\alpha, \beta=1}^{n} D_{\alpha}\left(\boldsymbol{A}^{\alpha \beta}(x, t) D_{\beta} \boldsymbol{u}\right)=0,
$$

where $\boldsymbol{u}=\left(u^{1}, \ldots, u^{N}\right)^{T}$. We will make frequent use of a shorthand notation

$$
\left\langle\boldsymbol{A}^{\alpha \beta}(x, t) \boldsymbol{\xi}_{\beta}, \boldsymbol{\eta}_{\alpha}\right\rangle:=\sum_{\alpha, \beta=1}^{n} \sum_{i, j=1}^{N} A_{i j}^{\alpha \beta}(x, t) \xi_{\beta}^{j} \eta_{\alpha}^{i},
$$

where $\boldsymbol{\xi}_{\beta}=\left(\xi_{\beta}^{1}, \ldots, \xi_{\beta}^{N}\right)^{T}$ and $\boldsymbol{\eta}_{\alpha}=\left(\eta_{\alpha}^{1}, \ldots, \eta_{\alpha}^{N}\right)^{T}$ for $\alpha, \beta=1, \ldots, n$.

2000 Mathematics Subject Classification. Primary: 35A08; Secondary: 35B45, $35 \mathrm{~K} 10$.

Key words. Gaussian estimates, fundamental solutions, parabolic systems, a priori estimates. 
We assume that the system (1.1) is strongly parabolic; i.e., there is a number $\nu>0$ such that

$$
\nu|\boldsymbol{\xi}|^{2} \leq\left\langle\boldsymbol{A}^{\alpha \beta}(x, t) \boldsymbol{\xi}_{\beta}, \boldsymbol{\xi}_{\alpha}\right\rangle .
$$

Here, we used the notation

$$
|\boldsymbol{\xi}|^{2}:=\sum_{\alpha=1}^{n}\left|\boldsymbol{\xi}_{\alpha}\right|^{2}=\sum_{\alpha=1}^{n} \sum_{i=1}^{N}\left(\xi_{\alpha}^{i}\right)^{2}
$$

We also assume that there is a number $M>0$ such that

$$
\left|\left\langle\boldsymbol{A}^{\alpha \beta}(x, t) \boldsymbol{\xi}_{\beta}, \boldsymbol{\eta}_{\alpha}\right\rangle\right| \leq M|\boldsymbol{\xi}||\boldsymbol{\eta}| .
$$

In this article, we study fundamental solutions of the systems of equations (1.2). By a fundamental solution (or a fundamental matrix) $\boldsymbol{\Gamma}(x, t ; y, s)$ to the system (1.2) we mean an $N \times N$ matrix of functions defined for $t>s$ which, as a function of $(x, t)$, satisfies (1.2) (i.e., each column is a solution of (1.2)), and is such that

$$
\lim _{t \downarrow s} \int_{\mathbb{R}^{n}} \boldsymbol{\Gamma}(x, t ; y, s) \boldsymbol{f}(y) d y=\boldsymbol{f}(x)
$$

for any bounded continuous function $\boldsymbol{f}=\left(f^{1}, \ldots, f^{N}\right)^{T}$.

The adjoint system of (1.2) is given by

$$
\mathcal{L}^{*} \boldsymbol{v}:=\boldsymbol{v}_{t}+\sum_{\alpha, \beta=1}^{n} D_{\alpha}\left({ }^{*} \boldsymbol{A}^{\alpha \beta}(x, t) D_{\beta} \boldsymbol{v}\right)=0
$$

where ${ }^{*} \boldsymbol{A}^{\alpha \beta}=\left(\boldsymbol{A}^{\beta \alpha}\right)^{T}$, i.e., the transpose of $\boldsymbol{A}^{\beta \alpha}$. Note that

$$
\iint \mathcal{L} \boldsymbol{u} \cdot \boldsymbol{v}+\boldsymbol{u} \cdot \mathcal{L}^{*} \boldsymbol{v}=0 .
$$

For $X=(x, t) \in \mathbb{R}^{n+1}$ and $r>0$ we denote

$$
\begin{aligned}
& Q_{r}(X):=B_{r}(x) \times\left(t-r^{2}, t\right), \\
& Q_{r}^{*}(X):=B_{r}(x) \times\left(t, t+r^{2}\right) .
\end{aligned}
$$


Definition 1.1. We say that the operators $\mathcal{L}$ and $\mathcal{L}^{*}$ satisfy the local boundedness properties if there exists a constant $B$ independent of $r$ and $Y$ such that for any $\boldsymbol{u}$ satisfying the system (1.2) in $Q_{2 r}(Y)$ and any $\boldsymbol{v}$ satisfying the adjoint system $(1.7)$ in $Q_{2 r}^{*}(Y)$ the following local boundedness properties hold:

$$
\begin{aligned}
& \sup _{Q_{r}(Y)}|\boldsymbol{u}| \leq B\left[\frac{1}{\left|Q_{2 r}(Y)\right|} \int_{Q_{2 r}(Y)}|\boldsymbol{u}|^{2}\right]^{1 / 2}, \\
& \sup _{Q_{r}^{*}(Y)}|\boldsymbol{v}| \leq B\left[\frac{1}{\left|Q_{2 r}^{*}(Y)\right|} \int_{Q_{2 r}^{*}(Y)}|\boldsymbol{v}|^{2}\right]^{1 / 2} .
\end{aligned}
$$

In the next theorem, we make the qualitative assumption that the coefficients $A_{i j}^{\alpha \beta}(x, t)$ are smooth; however we emphasize that all qualitative estimates are only allowed to depend on the dimension $n$, parabolicity constants $\nu, M$ and the local boundedness constant $B$ as appears in Definition 1.1.

Theorem 1.1. Suppose that $\mathcal{L}$ and $\mathcal{L}^{*}$ satisfies the local boundedness properties. Then, the fundamental solution $\boldsymbol{\Gamma}(x, t ; y, s)$ has an upper bound

$$
|\boldsymbol{\Gamma}(x, t ; y, s)| \leq K_{0}(t-s)^{-n / 2} \exp \left\{-\frac{k_{0}|x-y|^{2}}{t-s}\right\}
$$

where $|\boldsymbol{\Gamma}(x, t ; y, s)|$ denotes the operator norm of fundamental matrix $\boldsymbol{\Gamma}(x, t ; y, s)$. Here, $K_{0}=K_{0}(n, \nu, M, B)$ and $k_{0}=k_{0}(\nu, M)$.

Proof: We follow a technique as appears in [5], which is based on a method introduced by E. B. Davies in [3], [4]. Let $\psi$ be a Lipschitz function such that $|\nabla \psi| \leq \gamma$ with $\gamma \geq 0$ to be determined later.

Let $\mathcal{S}^{N}\left(\mathbb{R}^{n}\right)$ denote a function space whose elements are $N \times 1$ column vectors of functions from Schwartz test function space. Define an operator $P_{s \rightarrow t}^{\psi}$ on $\mathcal{S}^{N}\left(\mathbb{R}^{n}\right)$ for $t>s$ by setting

$$
P_{s \rightarrow t}^{\psi} \boldsymbol{f}(x)=\exp (\psi(x)) \int_{\mathbb{R}^{n}} \boldsymbol{\Gamma}(x, t ; y, s) \exp (-\psi(y)) \boldsymbol{f}(y) d y
$$


for $\boldsymbol{f} \in \mathcal{S}^{N}\left(\mathbb{R}^{n}\right)$. Then, $\boldsymbol{u}(x, t)=e^{-\psi(x)} P_{s \rightarrow t}^{\psi} \boldsymbol{f}(x)$ satisfies (1.2).

$$
\begin{aligned}
\frac{d}{d t}\left\|P_{s \rightarrow t}^{\psi} \boldsymbol{f}\right\|_{L^{2}\left(\mathbb{R}^{n}\right)}^{2} & =\frac{d}{d t} \int_{\mathbb{R}^{n}} e^{2 \psi(x)}|\boldsymbol{u}(x, t)|^{2} d x \\
& =2 \int e^{2 \psi} \boldsymbol{u} \cdot \boldsymbol{u}_{t} \\
& =2 \int e^{2 \psi} \boldsymbol{u} \cdot D_{\alpha}\left(\boldsymbol{A}^{\alpha \beta} D_{\beta} \boldsymbol{u}\right) \\
& =-2 \int\left\langle\boldsymbol{A}^{\alpha \beta} D_{\beta} \boldsymbol{u}, D_{\alpha}\left(e^{2 \psi} \boldsymbol{u}\right)\right\rangle \\
& =-2 \int e^{2 \psi}\left\langle\boldsymbol{A}^{\alpha \beta} D_{\beta} \boldsymbol{u}, D_{\alpha} \boldsymbol{u}\right\rangle \\
& \leq-2 \nu \int e^{2 \psi}\left\langle\boldsymbol{A}^{\alpha \beta} D_{\beta} \boldsymbol{u}, D_{\alpha} \psi \boldsymbol{u}\right\rangle \\
& \leq \frac{2 M^{2}}{\nu} \gamma^{2} \int e^{2 \psi}\left|4 \boldsymbol{u}^{2}=\frac{2 M^{2}}{\nu} \gamma^{2}\left\|e_{s \rightarrow t}^{\psi} \mid D\right\|_{L^{2}\left(\mathbb{R}^{n}\right)}^{2} .\right.
\end{aligned}
$$

Since $\lim _{t \downarrow s} P_{s \rightarrow t}^{\psi} \boldsymbol{f}(x)=\boldsymbol{f}(x)$, the above differential inequality implies (1.15) $\left\|P_{s \rightarrow t}^{\psi} \boldsymbol{f}\right\|_{L^{2}\left(\mathbb{R}^{n}\right)} \leq e^{\kappa \gamma^{2}(t-s)}\|\boldsymbol{f}\|_{L^{2}\left(\mathbb{R}^{n}\right)}$, where $\quad \kappa=M^{2} / \nu$.

The adjoint of $P_{s \rightarrow t}^{\psi}$ is given by

$$
\left(P_{s \rightarrow t}^{\psi}\right)^{*} \boldsymbol{g}(y)=\exp (-\psi(y)) \int_{\mathbb{R}^{n}} \boldsymbol{\Gamma}^{*}(y, s ; x, t) \exp (\psi(x)) \boldsymbol{g}(x) d x
$$

where

$$
\boldsymbol{\Gamma}^{*}(y, s ; x, t)=\boldsymbol{\Gamma}(x, t ; y, s)^{T} .
$$

Note that $\Gamma^{*}(y, s ; x, t)$ is the fundamental solution to the adjoint system (1.7) (eee e.g. [6, Chapter 9]). In particular, $\boldsymbol{v}(y, s):=e^{\psi(y)}\left(P_{s \rightarrow t}^{\psi}\right)^{*} \boldsymbol{g}(y)$ satisfies (1.7).

A similar computation shows

$$
\left\|\left(P_{s \rightarrow t}^{\psi}\right)^{*} \boldsymbol{g}\right\|_{L^{2}\left(\mathbb{R}^{n}\right)} \leq e^{\kappa \gamma^{2}(t-s)}\|\boldsymbol{g}\|_{L^{2}\left(\mathbb{R}^{n}\right)} .
$$

In particular, by setting $\psi \equiv 0$ so that $\gamma=0$, we have

$$
\left\|P_{s \rightarrow t}^{0} \boldsymbol{f}\right\|_{L^{2}\left(\mathbb{R}^{n}\right)} \leq\|\boldsymbol{f}\|_{L^{2}\left(\mathbb{R}^{n}\right)}
$$


and

$$
\left\|\left(P_{s \rightarrow t}^{0}\right)^{*} \boldsymbol{g}\right\|_{L^{2}\left(\mathbb{R}^{n}\right)} \leq\|\boldsymbol{g}\|_{L^{2}\left(\mathbb{R}^{n}\right)} .
$$

By setting $\boldsymbol{u}(x, t):=P_{s \rightarrow t}^{0} \boldsymbol{f}(x)$ and using property (1.11) with $Y=(x, t)$ and $r=\sqrt{t-s} / 2$ we obtain

$$
\begin{aligned}
|\boldsymbol{u}(x, t)|^{2} & \leq \frac{B^{2}}{\omega_{n}(t-s)^{1+n / 2}} \int_{s}^{t} \int_{B_{\sqrt{t-s}}(x)}|\boldsymbol{u}(y, \tau)|^{2} d y d \tau \\
& \leq \frac{B^{2}}{\omega_{n}(t-s)^{n / 2}}\|\boldsymbol{f}\|_{L^{2}\left(\mathbb{R}^{n}\right)}^{2}
\end{aligned}
$$

Here, $\omega_{n}$ denotes the volume of unit ball $\left|B_{1}\right|$ in $\mathbb{R}^{n}$. We have thus derived the following $L^{2} \rightarrow L^{\infty}$ estimate for $P_{s \rightarrow t}^{0}$,

$$
\left\|P_{s \rightarrow t}^{0} \boldsymbol{f}\right\|_{L^{\infty}\left(\mathbb{R}^{n}\right)} \leq N_{0}(t-s)^{-n / 4}\|\boldsymbol{f}\|_{L^{2}\left(\mathbb{R}^{n}\right)}, \quad N_{0}=\sqrt{B^{2} / \omega_{n}}
$$

and similarly for $\left(P_{s \rightarrow t}^{0}\right)^{*}$

$$
\left\|\left(P_{s \rightarrow t}^{0}\right)^{*} \boldsymbol{f}\right\|_{L^{\infty}} \leq N_{0}(t-s)^{-n / 4}\|\boldsymbol{f}\|_{L^{2}} .
$$

In the case when $\gamma>0$, i.e., $\psi \not \equiv$ const., we set $\boldsymbol{u}(x, t):=e^{-\psi(x)} P_{s \rightarrow t}^{\psi} \boldsymbol{f}(x)$ and carry out a similar estimate to get

$$
\begin{aligned}
e^{-2 \psi(x)}\left|P_{s \rightarrow t}^{\psi} \boldsymbol{f}(x)\right|^{2} & =|\boldsymbol{u}(x, t)|^{2} \\
& \leq \frac{B^{2}}{\omega_{n}(t-s)^{1+n / 2}} \int_{s}^{t} \int_{B_{\sqrt{t-s}}(x)}|\boldsymbol{u}(y, \tau)|^{2} d y d \tau \\
& \leq \frac{B^{2}}{\omega_{n}(t-s)^{1+n / 2}} \int_{s}^{t} \int_{B_{\sqrt{t-s}}(x)} e^{-2 \psi(y)}\left|P_{s \rightarrow \tau}^{\psi} \boldsymbol{f}(y)\right|^{2} d y d \tau
\end{aligned}
$$

Hence

$$
\begin{aligned}
\left|P_{s \rightarrow t}^{\psi} \boldsymbol{f}(x)\right|^{2} & \leq \frac{B^{2}}{\omega_{n}(t-s)^{1+n / 2}} \int_{s}^{t} \int_{B_{\sqrt{t-s}}(x)} e^{2 \psi(x)-2 \psi(y)}\left|P_{s \rightarrow \tau}^{\psi} \boldsymbol{f}(y)\right|^{2} d y d \tau \\
& \leq \frac{B^{2}}{\omega_{n}(t-s)^{1+n / 2}} \int_{s}^{t} \int_{B_{\sqrt{t-s}}(x)} e^{2 \gamma \sqrt{t-s}}\left|P_{s \rightarrow \tau}^{\psi} \boldsymbol{f}(y)\right|^{2} d y d \tau \\
& \leq \frac{B^{2} e^{2 \gamma \sqrt{t-s}}}{\omega_{n}(t-s)^{1+n / 2}} \int_{s}^{t} e^{2 \kappa \gamma^{2}(\tau-s)}\|\boldsymbol{f}\|_{L^{2}\left(\mathbb{R}^{n}\right)}^{2} d \tau \\
& \leq \frac{B^{2} e^{2 \gamma \sqrt{t-s}}}{\omega_{n}(t-s)^{1+n / 2}} \frac{e^{2 \kappa \gamma^{2}(t-s)}}{2 \kappa \gamma^{2}}\|\boldsymbol{f}\|_{L^{2}\left(\mathbb{R}^{n}\right)}^{2} .
\end{aligned}
$$


Hence, the following $L^{2} \rightarrow L^{\infty}$ estimate for $P_{s \rightarrow t}^{\psi}$ follows for $\gamma>0$.

$$
\left\|P_{s \rightarrow t}^{\psi} \boldsymbol{f}\right\|_{L^{\infty}} \leq N_{1} \frac{\exp \left\{\gamma \sqrt{t-s}+\kappa \gamma^{2}(t-s)\right\}}{\gamma(t-s)^{1 / 2+n / 4}}\|\boldsymbol{f}\|_{L^{2}},
$$

where $N_{1}=\sqrt{B^{2} / 2 \omega_{n} \kappa}$. By a similar argument, we also have

$$
\left\|\left(P_{s \rightarrow t}^{\psi}\right)^{*} \boldsymbol{g}\right\|_{L^{\infty}} \leq N_{1} \frac{\exp \left\{\gamma \sqrt{t-s}+\kappa \gamma^{2}(t-s)\right\}}{\gamma(t-s)^{1 / 2+n / 4}}\|\boldsymbol{g}\|_{L^{2}} .
$$

By duality, (1.22) and (1.24) imply $L^{1} \rightarrow L^{2}$ estimates

$$
\begin{aligned}
& \left\|P_{s \rightarrow t}^{0} \boldsymbol{f}\right\|_{L^{2}} \leq N_{0}(t-s)^{-n / 4}\|\boldsymbol{f}\|_{L^{1}}, \\
& \left\|P_{s \rightarrow t}^{\psi} \boldsymbol{f}\right\|_{L^{2}} \leq N_{1} \frac{\exp \left\{\gamma \sqrt{t-s}+\kappa \gamma^{2}(t-s)\right\}}{\gamma(t-s)^{1 / 2+n / 4}}\|\boldsymbol{f}\|_{L^{1}} .
\end{aligned}
$$

Now, let $r=(s+t) / 2$. Observe that

$$
P_{s \rightarrow t}^{\psi}=P_{r \rightarrow t}^{\psi} \circ P_{s \rightarrow r}^{\psi} .
$$

By noting that $t-r=r-s=(t-s) / 2$, we have the following $L^{1} \rightarrow L^{\infty}$ estimates:

$$
\begin{aligned}
& \left\|P_{s \rightarrow t}^{0} \boldsymbol{f}\right\|_{L^{\infty}} \leq A_{0}(t-s)^{-n / 2}\|\boldsymbol{f}\|_{L^{1}}, \\
& \left\|P_{s \rightarrow t}^{\psi} \boldsymbol{f}\right\|_{L^{\infty}} \leq A_{1} \frac{\exp \left\{\gamma \sqrt{2(t-s)}+\kappa \gamma^{2}(t-s)\right\}}{\gamma^{2}(t-s)^{1+n / 2}}\|\boldsymbol{f}\|_{L^{1}\left(\mathbb{R}^{n}\right)},
\end{aligned}
$$

where $A_{0}=2^{n / 2} N_{0}^{2}$ and $A_{1}=2^{1+n / 2} N_{1}^{2}$.

For fixed $x, y \in \mathbb{R}^{n}(x \neq y)$, the above estimate (1.29) implies

$$
e^{\psi(x)-\psi(y)}|\boldsymbol{\Gamma}(x, t ; y, s)| \leq A_{1} \frac{\exp \left\{\gamma \sqrt{2(t-s)}+\kappa \gamma^{2}(t-s)\right\}}{\gamma^{2}(t-s)^{1+n / 2}} .
$$

Let $\psi(z)=\gamma|z-y|$. Then $\psi$ is Lipschitz function with $|\nabla \psi| \leq \gamma$. With this choice of $\psi$, it follows from (1.30)

$$
|\boldsymbol{\Gamma}(x, t ; y, s)| \leq A_{1} \frac{\exp \left(\gamma \sqrt{2(t-s)}+\kappa \gamma^{2}(t-s)-\gamma|x-y|\right)}{\gamma^{2}(t-s)^{1+n / 2}} .
$$

Now if we set $\gamma=|x-y| / 2 \kappa(t-s)$ and $\xi=|x-y| / \sqrt{t-s}$,

$$
|\boldsymbol{\Gamma}(x, t ; y, s)| \leq \frac{4 A_{1} \kappa^{2}}{(t-s)^{n / 2}} \frac{\exp \left(\xi / \sqrt{2} \kappa-\xi^{2} / 4 \kappa\right)}{\xi^{2}} .
$$

On the other hand, (1.28) gives an upper bound for $|\boldsymbol{\Gamma}(x, t ; y, s)|$ independent of $x$ and $y$,

$$
|\boldsymbol{\Gamma}(x, t ; y, s)| \leq A_{0}(t-s)^{-n / 2} .
$$


Combining (1.32) and (1.33) together,

$$
|\boldsymbol{\Gamma}(x, t ; y, s)| \leq \frac{2^{n / 2} B^{2}}{\omega_{n}(t-s)^{n / 2}} \min \left(1,4 \kappa \frac{\exp \left(\xi / \sqrt{2} \kappa-\xi^{2} / 4 \kappa\right)}{\xi^{2}}\right)
$$

Choose $R=R(\kappa)=R\left(M^{2} / \nu\right)$ so that

$$
\frac{\exp \left(\xi / \sqrt{2} \kappa-\xi^{2} / 4 \kappa\right)}{\xi^{2}} \leq \frac{1}{4 \kappa} \exp \left(-\xi^{2} / 8 \kappa\right), \quad \forall \xi>R
$$

Now set $k_{0}=1 / 8 \kappa=M^{2} / 8 \nu$ and and $K_{0}=\left(2^{n / 2} B^{2} / \omega_{n}\right) \exp \left(M^{2} R^{2} / 8 \nu\right)$. Then, we have the desired estimate (1.13). The proof is complete.

Remark 1.1. If $A_{i j}^{\alpha \beta}$ is symmetric, i.e., if $A_{i j}^{\alpha \beta}=A_{j i}^{\beta \alpha}$, then the local boundedness property $(1.12)$ for $\mathcal{L}^{*}$ follows from the local boundedness property (1.11) for $\mathcal{L}$.

To see this, let $\boldsymbol{v}$ be a solution to $\mathcal{L}^{*} \boldsymbol{v}=0$ in $Q_{2 r}^{*}(Y)$ and denote $Y^{*}=(y,-s)$. Since ${ }^{*} \boldsymbol{A}^{\alpha \beta}=\boldsymbol{A}^{\alpha \beta}, \tilde{\boldsymbol{u}}(x, t):=\boldsymbol{v}(x,-t)$ satisfies $\mathcal{L} \tilde{\boldsymbol{u}}=0$ in $Q_{2 r}\left(Y^{*}\right)$ and thus satisfies (1.11) by the assumption. Therefore $\boldsymbol{v}$ satisfies (1.12).

The following theorem is the converse of Theorem 1.1. Here, we also make the qualitative assumption that the coefficients $A_{i j}^{\alpha \beta}(x, t)$ are smooth.

Theorem 1.2. Assume that the fundamental solution $\boldsymbol{\Gamma}$ has an upper bound (1.13). Then, any solution $\boldsymbol{u}$ to (1.2) in $Q_{4 R}=Q_{4 R}\left(x_{0}, t_{0}\right)$ satisfies the local boundedness property

$$
\sup _{Q_{R}}|\boldsymbol{u}| \leq B\left[\frac{1}{\left|Q_{4 R}\right|} \int_{Q_{4 R}}|\boldsymbol{u}|^{2}\right]^{1 / 2}
$$

with $B=B\left(n, N, k_{0}, K_{0}, \nu, M\right)$.

Proof: Let $\zeta$ be a smooth cut-off function such that $\zeta \equiv 1$ on $Q_{2 R}$, $\zeta \equiv 0$ outside $Q_{3 R},\left|\zeta_{t}\right| \leq \frac{4}{R^{2}}$, and $\left|\nabla_{x} \zeta\right| \leq \frac{2}{R}$. Then $\boldsymbol{v}:=\zeta \boldsymbol{u}$ satisfies

(1.36) $\boldsymbol{v}_{t}-D_{\alpha}\left(\boldsymbol{A}^{\alpha \beta} D_{\beta} \boldsymbol{v}\right)=\zeta_{t} \boldsymbol{u}-D_{\alpha}\left(\boldsymbol{A}^{\alpha \beta} D_{\beta} \zeta \boldsymbol{u}\right)-\boldsymbol{A}^{\alpha \beta} D_{\alpha} \zeta D_{\beta} \boldsymbol{u}=: \boldsymbol{f}$ 
We extend $\boldsymbol{\Gamma}(x, t ; y, s) \equiv 0$ for $t<s$. Let $X=(x, t) \in Q_{R}$ be fixed and denote $\boldsymbol{\Gamma}^{X}(y, s)=\boldsymbol{\Gamma}(x, t ; y, s)$. By Duhamel's principle (see e.g. [6, Chapter 9]), we get from (1.36)

$$
\begin{aligned}
\boldsymbol{u}(x, t) & =\int_{-\infty}^{t} \int_{\mathbb{R}^{n}} \boldsymbol{\Gamma}(x, t ; y, s) \boldsymbol{f}(y, s) d y d s \\
& =\iint_{s<t} \boldsymbol{\Gamma}^{X} \zeta_{s} \boldsymbol{u}-\iint_{s<t} \boldsymbol{\Gamma}^{X} D_{\alpha}\left(\boldsymbol{A}^{\alpha} D_{\beta} \zeta \boldsymbol{u}\right)-\iint_{s<t} \boldsymbol{\Gamma}^{X} \boldsymbol{A}^{\alpha \beta} D_{\alpha} \zeta D_{\beta} \boldsymbol{u} \\
& =: I+I I+I I I .
\end{aligned}
$$

Denote $Q=\left(Q_{3 R} \backslash Q_{2 R}\right) \cap\{s<t\}$. We estimate

$$
|I| \leq \frac{4}{R^{2}}\left(\int_{Q}|\boldsymbol{\Gamma}(x, t ; y, s)|^{2} d y d s\right)^{1 / 2}\left(\int_{Q_{4 R}}|\boldsymbol{u}|^{2}\right)^{1 / 2} .
$$

Note that we have localized energy inequality (see e.g. [11])

$$
\int_{Q_{3 R}}|D \boldsymbol{u}|^{2} \leq \frac{N_{0}}{R^{2}} \int_{Q_{4 R}}|\boldsymbol{u}|^{2},
$$

where $N_{0}=N_{0}(\nu, M)$. Using this we estimate

$$
\begin{aligned}
|I I I| & \leq \frac{2 M}{R}\left(\int_{Q}|\boldsymbol{\Gamma}(x, t ; y, s)|^{2} d y d s\right)^{1 / 2}\left(\int_{Q_{3 R}}|D \boldsymbol{u}|^{2}\right)^{1 / 2} \\
& \leq \frac{N_{1}}{R^{2}}\left(\int_{Q}|\boldsymbol{\Gamma}(x, t ; y, s)|^{2} d y d s\right)^{1 / 2}\left(\int_{Q_{4 R}}|\boldsymbol{u}|^{2}\right)^{1 / 2} .
\end{aligned}
$$

Note that the transpose of $\boldsymbol{\Gamma}(x, t ; y, s)$ is $\boldsymbol{\Gamma}^{*}(y, s ; x, t)$, the fundamental solution to the adjoint system.

$$
\begin{aligned}
I I & =\int_{-\infty}^{t} \int_{\mathbb{R}^{n}} D_{y_{\alpha}} \boldsymbol{\Gamma}(x, t ; y, s) \boldsymbol{A}^{\alpha \beta} D_{\beta} \zeta \boldsymbol{u} \\
& =\int_{-\infty}^{t} \int_{\mathbb{R}^{n}}\left[D_{y_{\alpha}} \boldsymbol{\Gamma}^{*}(y, s ; x, t)\right]^{T} \boldsymbol{A}^{\alpha \beta} D_{\beta} \zeta \boldsymbol{u}(y, s) d y d s .
\end{aligned}
$$

Therefore, we estimate

$$
|I I| \leq \frac{2 M}{R}\left(\int_{Q}\left|D_{y} \Gamma^{*}(y, s ; x, t)\right|^{2} d y d s\right)^{1 / 2}\left(\int_{Q_{4 R}}|\boldsymbol{u}|^{2}\right)^{1 / 2}
$$


Note that $\boldsymbol{\Gamma}^{*}(y, s ; x, t)$ satisfies the adjoint system (1.7) in $\{s<t\}$. More precisely, each column vector $\boldsymbol{w}(y, s)$ of $\boldsymbol{\Gamma}^{*}(y, s ; x, t)$ satisfies

$$
\boldsymbol{w}_{s}+D_{\alpha}\left({ }^{*} A^{\alpha \beta}(y, s) D_{\beta} \boldsymbol{w}\right)=0 .
$$

Let $\eta$ be a smooth cut-off function such that $\eta \equiv 1$ on $Q_{4 R}(X) \backslash Q_{R}(X)$, $\eta \equiv 0$ on $Q_{R / 2}(X)$ and outside $Q_{5 R}(X)$, and $\left|D_{s} \eta\right|+\left|\nabla_{y} \eta\right|^{2} \leq \frac{100}{R^{2}}$. In particular, note that $\eta \equiv 1$ on $Q$.

Note that $\lim _{s \uparrow t} \eta^{2}|\boldsymbol{w}|^{2}(\cdot, s) \equiv 0$ by the choice of $\eta$ and by (1.13). Therefore, as it is done in the proof of localized energy estimates we have

$$
\int_{Q}|D \boldsymbol{w}|^{2} \leq \frac{N_{2}}{R^{2}} \int_{Q^{\prime}}|\boldsymbol{w}|^{2}, \quad \text { where } \quad Q^{\prime}:=Q_{5 R}(X) \backslash Q_{R / 2}(X) .
$$

Therefore, by the equivalence of norms in finite dimensional vector spaces,

$$
\int_{Q}\left|D_{y} \boldsymbol{\Gamma}^{*}(y, s ; x, t)\right|^{2} d y d s \leq \frac{N_{3}}{R^{2}} \int_{Q^{\prime}}|\boldsymbol{\Gamma}(x, t ; y, s)|^{2} d y d s,
$$

and hence together with (1.39), we get

$$
|I I| \leq \frac{N_{4}}{R^{2}}\left(\int_{Q^{\prime}}|\boldsymbol{\Gamma}(x, t ; y, s)|^{2} d y d s\right)^{1 / 2}\left(\int_{Q_{4 R}}|\boldsymbol{u}|^{2}\right)^{1 / 2} .
$$

Since $Q \subset Q^{\prime}$ we have

$$
\begin{aligned}
|\boldsymbol{u}(x, t)| & \leq|I|+|I I|+|I I I| \\
& \leq \frac{N_{5}}{R^{2}}\left(\int_{Q^{\prime}}|\boldsymbol{\Gamma}(x, t ; y, s)|^{2} d y d s\right)^{1 / 2}\left(\int_{Q_{4 R}}|\boldsymbol{u}|^{2}\right)^{1 / 2} .
\end{aligned}
$$

We claim that the following estimate holds.

$$
\int_{Q^{\prime}}|\boldsymbol{\Gamma}(x, t ; y, s)|^{2} d y d s \leq A_{0} R^{2-n}, \quad A_{0}=A_{0}\left(n, k_{0}, K_{0}\right) .
$$

Then the estimate (1.35) follows from (1.43).

It remains to prove the claim (1.44). In the view of (1.13) and the definition $Q^{\prime}=Q_{5 R}(X) \backslash Q_{R / 2}(X)$, it suffices to show the following estimates:

$$
\begin{array}{r}
\int_{0}^{(R / 2)^{2}} \int_{R / 2}^{\infty}+\int_{(R / 2)^{2}}^{(5 R)^{2}} \int_{0}^{\infty} n \omega_{n} K_{0}^{2} s^{-n} e^{-2 k_{0} r^{2} / s} r^{n-1} d r d s \\
=: I V+V \leq A_{0} R^{2-n}
\end{array}
$$


Using Fubini's theorem and change of variable,

$$
I V=\frac{n \omega_{n} K_{0}^{2}}{\left(2 k_{0}\right)^{n-1}} \int_{R / 2}^{\infty} r^{1-n} \int_{8 k_{0} r^{2} / R^{2}}^{\infty} e^{-u} u^{n-2} d u d r .
$$

A straightforward computation will show that the integral in (1.45) is bounded by $A_{0} R^{2-n}$ for some $A_{0}=A_{0}\left(n, k_{0}, K_{0}\right)$. For example, if $n \geq 3$, we estimate

$$
\int_{8 k_{0} r^{2} / R^{2}}^{\infty} e^{-u} u^{n-2} d u \leq \int_{0}^{\infty} e^{-u} u^{n-2} d u=(n-2) !
$$

so that

$$
I V \leq \frac{n \omega_{n} K_{0}^{2}(n-3) !}{2 k_{0}^{n-1}} R^{2-n} .
$$

Similarly, by a change of variable, we estimate

$$
V=\frac{n \omega_{n} K_{0}^{2}}{2\left(2 k_{0}\right)^{n / 2}} \int_{(R / 2)^{2}}^{(5 R)^{2}} s^{-n / 2} \int_{0}^{\infty} e^{-u} u^{n / 2-1} d u d s .
$$

Again, a straightforward calculation yields that $V \leq A_{0}\left(n, k_{0}, K_{0}\right) R^{2-n}$. For example, in the case when $n \geq 3$,

$$
V \leq \frac{n \omega_{n} K_{0}^{2} \Gamma(n / 2)}{2\left(2 k_{0}\right)^{n / 2}} \int_{(R / 2)^{2}}^{\infty} s^{-n / 2} d s=\frac{K_{0}^{2}(2 \pi)^{n / 2}}{2(n-2) k_{0}^{n / 2}} R^{2-n} .
$$

The proof is complete.

Remark 1.2. Assume that the fundamental solution $\boldsymbol{\Gamma}$ to the system (1.2) has an upper bound (1.13) and let $\boldsymbol{u}$ be a solution to (1.2) in $Q_{R}$. We can deduce the following estimates from (1.35). (see e.g. [8, pp. 80-81]).

(1) For any $\tau \in(0,1)$, we have

$$
\sup _{Q_{\tau R}}|\boldsymbol{u}| \leq \frac{B}{(1-\tau)^{\frac{n+2}{2}}}\left(\frac{1}{\left|Q_{R}\right|} \int_{Q_{R}}|\boldsymbol{u}|^{2}\right)^{1 / 2} .
$$

(2) For any $p>0$, there is a constant $c(p)$ such that

$$
\sup _{Q_{\rho}}|\boldsymbol{u}| \leq \frac{c(p)}{(R-\rho)^{\frac{n+2}{p}}}\left(\int_{Q_{R}}|\boldsymbol{u}|^{p}\right)^{1 / p} \quad \text { for all } \rho<R .
$$




\section{Applications}

In this section, we will give examples of parabolic systems satisfying the local boundedness properties and thus satisfying the Gaussian upper bound estimates. For example, "almost diagonal" parabolic systems belong to such classes. This fact can be deduced by using a well-known technique due to Campanato. (see e.g. [7]). We will provide a proof for the sake of completeness.

Let us first recall some well-known facts. The following lemma is N. G. Meyers' integral characterization of Hölder continuous functions. See e.g. [13] and [12, Lemma 4.3, p. 50] for the proof.

Lemma 2.1. Let $\boldsymbol{u} \in L^{2}\left(Q_{2 R}\right)$ and suppose there are positive constants $\mu \leq 1$ and $H$ such that

$$
\int_{Q_{r}(X)}\left|\boldsymbol{u}-\boldsymbol{u}_{X, r}\right|^{2} \leq H^{2} r^{n+2+2 \mu}
$$

for any $X \in Q_{R}$ and any $r \in(0, R)$. Here $\boldsymbol{u}_{X, r}$ denotes the average of $\boldsymbol{u}$ over $Q_{r}(X)$. Then $\boldsymbol{u}$ is Hölder continuous with the exponent $\mu$ in $Q_{R}$ and $[\boldsymbol{u}]_{\mu, \mu / 2 ; Q_{R}} \leq N(n, \mu) H$.

The following lemma is found in [7, Lemma 2.1, p. 86].

Lemma 2.2. Let $\phi(t)$ be a nonnegative and nondecreasing function. Suppose that

$$
\phi(\rho) \leq A\left[\left(\frac{\rho}{r}\right)^{\sigma}+\varepsilon\right] \phi(r)+B r^{\tau}
$$

for all $\rho<r \leq R$, with $A, \sigma, \tau$ nonnegative constants, $\tau<\sigma$. Then there exists a constant $\varepsilon_{0}=\varepsilon_{0}(A, \sigma, \tau)$ such that if $\varepsilon<\varepsilon_{0}$, for all $\rho<r \leq R$ we have

$$
\phi(\rho) \leq c\left[\left(\frac{\rho}{r}\right)^{\tau} \phi(r)+B \rho^{\tau}\right]
$$

where $c$ is a constant depending on $\sigma, \tau, A$.

A proof of the following lemma can be found in [15], [9].

Lemma 2.3. There exists a constant $P_{0}=P_{0}(n, \nu, M)$ such that for any solution $\boldsymbol{u}$ to the system (1.2) in $Q_{R}$,

$$
\int_{Q_{R}}\left|\boldsymbol{u}-\boldsymbol{u}_{R}\right|^{2} \leq P_{0} R^{2} \int_{Q_{R}}|D \boldsymbol{u}|^{2} .
$$

Here, $\boldsymbol{u}_{R}$ denotes the average of $\boldsymbol{u}$ over $Q_{R}$. 
Proposition 2.1. Let $a(x, t)=\left[a^{\alpha \beta}(x, t)\right]$ be a real $n \times n$ matrix satisfying uniform parabolicity condition

$$
a^{\alpha \beta}(x, t) \xi_{\alpha} \xi_{\beta} \geq \lambda|\xi|^{2}, \quad \sum\left|a^{\alpha \beta}(x, t)\right|^{2} \leq \Lambda^{2} .
$$

There exists $\delta_{0}=\delta_{0}(n, \lambda, \Lambda)$ such that if

$$
\sum_{i, j=1}^{N} \sum_{\alpha, \beta=1}^{n}\left|A_{i j}^{\alpha \beta}-a^{\alpha \beta} \delta_{i j}\right|^{2}<\delta_{0}^{2},
$$

then local boundedness property holds for solutions to the system (1.2) associated with $A_{i j}^{\alpha \beta}$; i.e., there is a number $\delta_{0}$ such that if $A_{i j}^{\alpha \beta}$ satisfies (2.3), then for any solution $\boldsymbol{u}$ to (1.1) in $Q_{8 R}=Q_{8 R}\left(X_{0}\right)$ satisfies

$$
\sup _{Q_{R}}|\boldsymbol{u}| \leq B\left(\frac{1}{\left|Q_{8 R}\right|} \int_{Q_{8 R}}|\boldsymbol{u}|^{2}\right)^{1 / 2},
$$

for some $B=B(n, \lambda, \Lambda)$.

Proof: Note that the conditions (2.2) and (2.3) are invariant under the change of variables $x \mapsto\left(x-x_{0}\right) / R, t \mapsto\left(t-t_{0}\right) / R^{2}$. Thus, we may and do assume that $R=1$ and $X_{0}=0$.

Let $\boldsymbol{u}$ be a solution to (1.2) in $Q_{8}=Q_{8}(0)$. Fix $X \in Q_{2}$ and $0<r \leq 2$. Let $\boldsymbol{v}$ be a solution to

$$
\begin{cases}\boldsymbol{v}_{t}-D_{\alpha}\left(a^{\alpha \beta} D_{\beta} \boldsymbol{v}\right)=0 & \text { in } Q_{r}(X) \\ \boldsymbol{v}=\boldsymbol{u} & \text { on } \partial_{p} Q_{r}(X) .\end{cases}
$$

We apply Moser-Nash theory to each $v^{i}$ so that we have interior Hölder estimates, which, by a well-known argument, are equivalent to the following:

$$
\int_{Q_{\rho}(X)}|D \boldsymbol{v}|^{2} \leq C_{0}\left(\frac{\rho}{r}\right)^{n+2 \gamma} \int_{Q_{r}(X)}|D \boldsymbol{v}|^{2}, \quad 0<\rho \leq r,
$$

for some constants $C_{0}(n, \lambda, \Lambda)$ and $\gamma>0$. The point is that this Dirichlet integral characterization of Hölder continuity is stable under perturbation, as we shall demonstrate. We note that Auscher also exploits the stability of (2.5) (or to be more precise, of its elliptic analogue) in his work on Gaussian bounds [1].

Note that $\boldsymbol{w}:=\boldsymbol{u}-\boldsymbol{v}$ satisfies

$$
\begin{cases}\boldsymbol{w}_{t}-D_{\alpha}\left(a^{\alpha \beta} D_{\beta} \boldsymbol{w}\right)=D_{\alpha}\left(\tilde{\boldsymbol{A}}^{\alpha \beta} D_{\beta} \boldsymbol{u}\right) & \text { in } Q_{r}(X), \\ \boldsymbol{w}=0 & \text { on } \partial_{p} Q_{r}(X),\end{cases}
$$

where $\tilde{\boldsymbol{A}}^{\alpha \beta}:=\boldsymbol{A}^{\alpha \beta}-a^{\alpha \beta} I$. 
Since $\boldsymbol{w}=0$ on $\partial_{p} Q_{r}(X)$, we may use $\boldsymbol{w}$ itself as a test function to $(2.6)$ and get

$$
\int_{Q_{r}(X)}|D \boldsymbol{w}|^{2} \leq C_{1}\|\tilde{\boldsymbol{A}}\|_{\infty} \int_{Q_{r}(X)}|D \boldsymbol{u}|^{2}
$$

where

$$
\|\tilde{\boldsymbol{A}}\|_{\infty}^{2}:=\sup _{(x, t)} \sum_{i, j=1}^{N} \sum_{\alpha, \beta=1}^{n}\left|A_{i j}^{\alpha \beta}(x, t)-a^{\alpha \beta}(x, t) \delta_{i j}\right|^{2} .
$$

Therefore, we have

(2.9) $\int_{Q_{\rho}(X)}|D \boldsymbol{u}|^{2} \leq C_{2}\left(\frac{\rho}{r}\right)^{n+2 \gamma} \int_{Q_{r}(X)}|D \boldsymbol{u}|^{2}+C_{2}\|\tilde{\boldsymbol{A}}\|_{\infty}^{2} \int_{Q_{r}(X)}|D \boldsymbol{u}|^{2}$.

Now we choose $\delta_{0}$ so that $C_{2}\|\tilde{\boldsymbol{A}}\|_{\infty}^{2}<C_{2} \delta_{0}^{2}<\varepsilon_{0}$, where $\varepsilon_{0}$ is as in Lemma 2.2. Then, by Lemma 2.2 we have

$$
\int_{Q_{\rho}(X)}|D \boldsymbol{u}|^{2} \leq C_{3}\left(\frac{\rho}{r}\right)^{n+2 \mu} \int_{Q_{r}(X)}|D \boldsymbol{u}|^{2},
$$

where $\mu<\gamma$ (e.g. we may choose $\mu=\gamma / 2$ ).

By replacing $\delta_{0}$ by a smaller number if necessary, we may assume that $A_{i j}^{\alpha \beta}$ satisfy the parabolicity conditions (1.4) and (1.5) with $\nu=\lambda / 2$ and $M=2 \Lambda$ (see proof of the following Theorem 2.1). Then, it is easy to see that $\boldsymbol{u}$ satisfies the Caccioppoli's inequality

$$
\int_{Q_{r}(X)}|D \boldsymbol{u}|^{2} \leq \frac{N_{0}}{r^{2}} \int_{Q_{2 r}(X)}|\boldsymbol{u}|^{2} .
$$

By Lemma 2.3 and (2.11), we get, for all $0<\rho<r \leq 2$,

$$
\int_{Q_{\rho}(X)}\left|\boldsymbol{u}-\boldsymbol{u}_{\rho}\right|^{2} \leq N_{1}\left(\frac{\rho}{r}\right)^{n+2+2 \mu} \int_{Q_{2 r(X)}}|\boldsymbol{u}|^{2} .
$$

In particular, by setting $r=2$ and noticing $Q_{4}(X) \subset Q_{8}$, we conclude

$$
\int_{Q_{\rho}(X)}\left|\boldsymbol{u}-\boldsymbol{u}_{\rho}\right|^{2} \leq N_{2} \rho^{n+2+2 \mu} \int_{Q_{8}}|\boldsymbol{u}|^{2} .
$$

Therefore, by Lemma 2.1, we have

$$
[\boldsymbol{u}]_{\mu, \mu / 2 ; Q_{2}} \leq N_{3}\|\boldsymbol{u}\|_{L^{2}\left(Q_{8}\right)} .
$$

Now, let $X$ be a point in $Q_{1}$. Then, for all $Y \in Q_{1}(X) \subset Q_{2}$, we have (2.15) $\quad|\boldsymbol{u}(X)| \leq|\boldsymbol{u}(Y)|+|\boldsymbol{u}(X)-\boldsymbol{u}(Y)| \leq|\boldsymbol{u}(Y)|+[\boldsymbol{u}]_{\mu, \mu / 2 ; Q_{1}(X)}$. Integrating (2.15) over $Q_{1}(X)$ and using Hölder's inequality we get

$$
|\boldsymbol{u}(X)| \leq c(n)\left(\|\boldsymbol{u}\|_{L^{2}\left(Q_{2}\right)}+[\boldsymbol{u}]_{\mu, \mu / 2 ; Q_{2}}\right) \quad \forall X \in Q_{1} .
$$


Hence, (2.16) and (2.14) yields

$$
\sup _{Q_{1}}|\boldsymbol{u}| \leq B\|\boldsymbol{u}\|_{L^{2}\left(Q_{8}\right)} .
$$

The proof is complete.

Remark 2.1. Let $\mathcal{L}$ be an operator satisfying the hypothesis of Proposition 2.1. By Remark 1.2, we see that (2.4) implies the local boundedness property (1.11) for $\mathcal{L}$. In fact, in this case $\mathcal{L}^{*}$ also enjoys the local boundedness property (1.12).

Let $\boldsymbol{v}$ be a solution to the corresponding adjoint system in $Q_{2 r}^{*}(Y)$. Denote $\tilde{a}(x, t):=a(x,-t)^{T}$ and $\tilde{\boldsymbol{A}}^{\alpha \beta}(x, t):=\boldsymbol{A}^{\beta \alpha}(x,-t)^{T}$. Since the hypothesis of Proposition 2.1 remains the same for $\tilde{a}$ and $\tilde{\boldsymbol{A}}^{\alpha \beta}$, we find $\tilde{\boldsymbol{u}}(x, t):=\boldsymbol{v}(x,-t)$ is a solution to the system

$$
\tilde{\boldsymbol{u}}_{t}-\sum_{\alpha, \beta=1}^{n} D_{\alpha}\left(\tilde{\boldsymbol{A}}^{\alpha \beta}(x, t) D_{\beta} \tilde{\boldsymbol{u}}\right)=0
$$

in $Q_{2 r}\left(Y^{*}\right)$ where $Y^{*}=(y,-s)$. Therefore, we see that $\tilde{\boldsymbol{u}}$ satisfies the estimate (1.11) in $Q_{2 r}\left(Y^{*}\right)$, and thus $\boldsymbol{v}$ satisfies the estimate (1.12) in $Q_{2 r}^{*}(Y)$.

Theorem 2.1. Let $a_{0}(x, t)=\left[a_{0}^{\alpha \beta}(x, t)\right]$ be a real $n \times n$ matrix satisfying uniform parabolicity condition (2.2). Then there exists $\delta_{0}$ depending on dimension $n$ and constants $\lambda, \Lambda$ only, such that if a $(x, t)$ has complexvalued coefficients and $\left\|a-a_{0}\right\|_{\infty}<\delta_{0}$ then the fundamental solution $\Gamma$ of the operator $L_{a}=\partial_{t}-\operatorname{Div}(a \nabla)$ has an Gaussian upper bound (1.13) with constants $K_{0}, k_{0}$ depending only on $n, \lambda, \Lambda$.

Proof: We shall make the qualitative assumption that the coefficients $a(x, t)$ are smooth. However, this assumption is dropped later in proof. Define $A_{i j}^{\alpha \beta}$ by $(i, j=1,2)$

$$
\boldsymbol{A}^{\alpha \beta}=\left(\begin{array}{rr}
\Re a^{\alpha \beta} & -\Im a^{\alpha \beta} \\
\Im a^{\alpha \beta} & \Re a^{\alpha \beta}
\end{array}\right) .
$$

If $\delta_{0}$ is small enough, $A_{i j}^{\alpha \beta}(i, j=1,2)$ satisfies (1.4) and (1.5). Indeed, if we set $\tilde{\boldsymbol{A}}^{\alpha \beta}=\boldsymbol{A}^{\alpha \beta}-a_{0}^{\alpha \beta} I$, then $\|\tilde{\boldsymbol{A}}\|_{\infty}=\sqrt{2}\left\|a-a_{0}\right\|_{\infty}<\sqrt{2} \delta_{0}$, where $\|\tilde{\boldsymbol{A}}\|_{\infty}$ is defined as in (2.8), and

$$
A_{i j}^{\alpha \beta} \xi_{\alpha}^{i} \eta_{\beta}^{j}=a_{0}^{\alpha \beta} \xi_{\alpha}^{1} \eta_{\beta}^{1}+a_{0}^{\alpha \beta} \xi_{\alpha}^{2} \eta_{\beta}^{2}+\tilde{A}_{i j}^{\alpha \beta} \xi_{\alpha}^{i} \eta_{\beta}^{j} .
$$

Hence, we have

$$
\left(\lambda-\sqrt{2} \delta_{0}\right)|\boldsymbol{\xi}|^{2} \leq\left\langle\boldsymbol{A}^{\alpha \beta} \boldsymbol{\xi}_{\alpha}, \boldsymbol{\xi}_{\beta}\right\rangle
$$


and

$$
\left|\left\langle\boldsymbol{A}^{\alpha \beta} \boldsymbol{\xi}_{\alpha}, \boldsymbol{\eta}_{\beta}\right\rangle\right| \leq\left(\Lambda+\sqrt{2} \delta_{0}\right)|\boldsymbol{\xi}||\boldsymbol{\eta}|
$$

Let $\boldsymbol{\Gamma}$ be the fundamental matrix to the parabolic system (1.1) whose coefficients $A_{i j}^{\alpha \beta}$ defined as in (2.19). By Proposition 2.1, Remark 2.1 and then by Theorem 1.1 we conclude that $\Gamma$ has an upper bound (1.13) with constants $K_{0}, k_{0}$ depending only on $n, \lambda, \Lambda$.

Observe that $u$ is a solution to $L_{a} u=0$ if and only if $\boldsymbol{u}=(\Re u, \Im u)$ is a solution to (1.1) with $A_{i j}^{\alpha \beta}$ defined as in (2.19). Also, note that $\boldsymbol{\Gamma}$ is given by

$$
\boldsymbol{\Gamma}=\left(\begin{array}{cc}
\Re \Gamma & -\Im \Gamma \\
\Im \Gamma & \Re \Gamma
\end{array}\right) .
$$

Therefore, we have $|\Gamma|=|\boldsymbol{\Gamma}|_{\text {op }}=|\boldsymbol{\Gamma}|$ and thus $|\Gamma|$ has an upper bound (1.13) with constants $K_{0}, k_{0}$ depending only on $n, \lambda, \Lambda$.

Note that in the proof of Proposition 2.1 we derived a uniform a-priori bound for Hölder norm for the solutions of $L_{a} u=0$. We also derived a uniform a-priori upper bound for the fundamental solution. Therefore, by a standard approximation argument (see e.g. [10, Chapter 2]), we are allowed to discard the extra smoothness assumptions on $a(x, t)$. The proof is complete.

\section{References}

[1] P. Auscher, Regularity theorems and heat kernel for elliptic operators, J. London Math. Soc. (2) 54(2) (1996), 284-296.

[2] S. Campanato, Equazioni paraboliche del secondo ordine e spazi $\mathcal{L}^{2, \theta}(\Omega, \delta)$, Ann. Mat. Pura Appl. (4) 73 (1966), 55-102.

[3] E. B. Davies, Explicit constants for Gaussian upper bounds on heat kernels, Amer. J. Math. 109(2) (1987), 319-333.

[4] E. B. DAvies, Heat kernel bounds for second order elliptic operators on Riemannian manifolds, Amer. J. Math. 109(3) (1987), $545-569$.

[5] E. B. FAbes And D. W. Stroock, A new proof of Moser's parabolic Harnack inequality using the old ideas of Nash, Arch. Rational Mech. Anal. 96(4) (1986), 327-338.

[6] A. Friedman, "Partial differential equations of parabolic type", Prentice-Hall, Inc., Englewood Cliffs, N.J., 1964.

[7] M. Giaquinta, "Multiple integrals in the calculus of variations and nonlinear elliptic systems", Annals of Mathematics Studies 105, Princeton University Press, Princeton, NJ, 1983. 
[8] M. Giaquinta, "Introduction to regularity theory for nonlinear elliptic systems", Lectures in Mathematics ETH Zrich, Birkhäuser Verlag, Basel, 1993.

[9] M. Giaquinta And M. Struwe, On the partial regularity of weak solutions of nonlinear parabolic systems, Math. Z. 179(4) (1982), $437-451$.

[10] V. A. Kondrat'ev and E. M. Landis, Qualitative theory of second-order linear partial differential equations, (Russian), in: "Partial differential equations, 3", (Russian), Itogi Nauki i Tekhniki, Akad. Nauk SSSR, Vsesoyuz. Inst. Nauchn. i Tekhn. Inform., Moscow, 1988, pp. 99-215, 220.

[11] O. A. Ladyženskaja, V. A. Solonnikov and N. N. Ural'Ce$\mathrm{VA}$, "Linear and quasilinear equations of parabolic type", (Russian), Translated from the Russian by S. Smith, Translations of Mathematical Monographs 23, American Mathematical Society, Providence, R.I., 1967.

[12] G. M. Lieberman, "Second order parabolic differential equations", World Scientific Publishing Co., Inc., River Edge, NJ, 1996.

[13] N. G. Meyers, Mean oscillation over cubes and Hölder continuity, Proc. Amer. Math. Soc. 15 (1964), 717-721.

[14] J. NASH, Continuity of solutions of parabolic and elliptic equations, Amer. J. Math. 80 (1958), 931-954.

[15] M. Struwe, On the Hölder continuity of bounded weak solutions of quasilinear parabolic systems, Manuscripta Math. 35(1-2) (1981), $125-145$.

Mathematics Department

University of Missouri

Columbia, Missouri 65211

USA

E-mail address: hofmann@math.missouri.edu

E-mail address: seick@math.missouri.edu

Primera versió rebuda el 4 de desembre de 2003, darrera versió rebuda el 27 d'abril de 2004 . 